\title{
De trocar sale trocante: creatividad léxica y diccionarios académicos*
}

\author{
MARIANO QUIRÓS GARCÍA \\ Instituto de Lengua, Literatura y Antropología (CCHS - CSIC)
}

\section{Planteamiento}

En contraposición a su empleo como fuente de información semántica e incluso ortográfica, es difícil determinar cuántos y qué tipo de hablantes se dirigen al diccionario en busca de pautas morfológicas (asunto bien distinto es que su inserción en dicho espacio resulte necesaria para fomentar un uso acertado de la lengua). La mayor parte de tales consultas, además, parece responder a dudas relacionadas con la flexión verbal o con el género y el número, particularmente en el caso de voces irregulares, motivo por el que la Real Academia Española decidió que en la vigésima segunda edición del Diccionario (DRAE-2001) dichos componentes debían recibir una particular atención. ${ }^{1}$

Por lo que se refiere a la morfología derivativa, la situación es bastante más delicada, puesto que, a pesar de algunas valiosas propuestas (Pena 2002), aún no existe consenso en cuanto a su imbricación con la (macro)estructura de un repertorio léxico de carácter general basado en el uso. De manera que, por ejemplo, tras la decisión de lematizar por primera vez en 1992 los principales sufijos de la lengua española con la intención de simplificar las etimologías de los vocablos derivados (DRAE-1992: VII), ${ }^{2} \mathrm{y}$ ante las expectativas o reacciones que pudo ocasionar tal medida, en el $D R A E-2001$ se advirtió que, dadas las posibilidades infinitas de formación de nuevas palabras mediante procedimientos de derivación y composición, en «este Diccionario solo aparecen aquellos términos que, vista la documentación de su empleo real, el Pleno académico ha decidido incluir» (DRAE-2001: XXXI). Es decir: por una parte, en un catálogo como el de la Academia no pueden tener cabida todas las contingencias, las probabilidades que subyacen a las propias reglas de formación de palabras (en adelante RFP), mientras

* La elaboración del presente trabajo ha sido posible gracias al contrato Ramón y Cajal que me fue concedido por el MCyT en la convocatoria 2004-2007, así como al proyecto de investigación HUM2006-11883-C04-02, financiado por el MEC.

${ }^{1}$ Como señalan Buenafuentes de la Mata y Sánchez Lancis (2006: 25-26), una de las principales novedades del DRAE-2001 fue la inclusión de un total de 684 marcas de ortografía y 931 de información morfológica. Según los cálculos ofrecidos en su trabajo, el $92.8 \%$ de estas últimas alude al modelo flexivo de verbos irregulares, mientras que el 7.2\% del total se dedica a la gradación del adjetivo, a la formación de los superlativos irregulares y al género de algunos sustantivos. Añádase a esto la posibilidad de conjugar cualquier forma verbal que se ofrece tanto en la edición electrónica de la obra, como en la versión disponible en la página web de la Academia.

${ }^{2}$ La historia lexicográfica de los prefijos y otros elementos compositivos es sustancialmente diferente, como muestra el trabajo de Torres Martínez (2006). Me sirvo del NTLLE para la consulta y comparación de las distintas ediciones del DRAE. 
que, por otro lado, los testimonios escritos avalan la inclusión de voces regulares y transparentes, tanto desde el punto de vista formal como semántico.

No obstante, algunos estudiosos (Seco 2003a) han puesto de manifiesto el particular concepto de autoridad que ya descollaba en el primer Diccionario patrocinado por la Corporación en el siglo XVIII. De tal manera, que desde entonces no es infrecuente encontrarse ante vocablos y acepciones inexistentes, resultado de erratas, malas lecturas y otros azarosos despistes, que habitualmente son reconocidos como fantasmas lexicográficos; otros cuyo escueto número de documentaciones parece negarles su pretendido carácter general y, por último, términos cuyas credenciales remiten a otros diccionarios, lo que tampoco garantiza su efectividad.

Entre estos dos parámetros, la creatividad léxica que consienten los patrones derivacionales y el uso real, se han debatido algunos términos que engrosaron en algún momento el $D R A E$ o que todavía figuran entre sus páginas, como baratador, baratón y baratista (Quirós García 2007), o trocante, cuyo estatus lingüístico debe ser replanteado a la luz de los datos que poseemos. Modificaciones que, dependiendo de cada palabra en cuestión, pueden reclamar su matización o su exclusión definitiva del que es considerado como léxico oficial de nuestro idioma.

\section{HISTORIA LEXICOGRÁFICA DE TROCANTE}

En 1803, en la cuarta edición del Diccionario académico, apareció recogida por vez primera la palabra trocante, de la que se ofrecía como información morfológica su condición de participio activo del verbo trocar y se definía como 'el que trueca'. Además, se consideraba desde el punto de vista diacrónico como una voz anticuada, adjetivo con el que se identificaba «lo que ha mucho tiempo que no está en uso. Dícese comúnmente de las leyes de una nación o de las voces y frases de alguna lengua». De modo que, aunque no se dedicara un espacio particular al significado y valor de ciertas marcas, ${ }^{3}$ podemos deducir que el vocablo en cuestión era ajeno al español de inicios del siglo XIX, momento en el que precisamente entró a formar parte del acervo lingüístico español.

Pero, como sucede en otras muchas ocasiones, la historia de este vocablo en el propio ámbito del $D R A E$ presenta algunas particularidades, unas más comprensibles que otras, o, si se prefiere, con mayor o menor fundamento. Así, en el $D R A E-1832$ se eliminó el infinitivo de la información morfológica, aunque se repuso en el $D R A E-1852$, y en el $D R A E-1884$, sin explicación alguna, se hizo desaparecer la marca que lo identificaba como un uso anticuado, situación que se mantuvo hasta el $D R A E$-1984 — en el $D R A E-1992$ la voz fue suprimida - y que se reiteró en las cuatro ediciones del Diccionario manual (1967-1989). Que desapareciera la información relativa al verbo base puede tener su parte de lógica, puesto que, aparte de ser de fácil deducción, trocante se hallaba precedido de palabras como trocable, trocadamente,

\footnotetext{
${ }^{3}$ En la siguiente edición, sin embargo, se advertía de que «se ha suprimido la calificación de raro y de poco usado, que no excluye a una palabra de ser parte legítima del lenguage común; y cuando las ha dejado sin uso su mala suerte, han pasado como debían a la clase de anticuadas» (DRAE-1817: Prólogo). Cuando en el DRAE-1925 se abordó su sistematización, se decidió que ant. indicaba «que la voz o la acepción pertenece exclusivamente al vocabulario de la Edad Media; pero también se califica de anticuada la forma de una palabra, como notomía por anatomía, que aunque usada hasta el siglo XVII, ha sido desechada en el lenguaje moderno» (DRAE-1925: Reglas para el uso de este diccionario).
} 
trocado, trocador, trocaico y trocamiento — todas, menos una, de la misma familia-, y estaba seguido de trocar, por lo que su etimología podía resultar más o menos transparente. En cambio, el prescindir de la marca cronológica tuvo unas consecuencias más trascendentales, ya que, en definitiva, entre 1884 y 1984 se le otorgó la categoría de palabra usual en el ámbito hispánico.

No encuentro ningún argumento filológico para esta última elisión, exceptuando el hecho de que, entre los lexicógrafos que siguieron el $D R A E$ y acogieron también trocante como voz patrimonial, Ramón Joaquín Domínguez fue el primero que en su Diccionario Nacionalo Gran Diccionario Clásico de la Lengua Española (1846-1847) la presentó sin etiquetado cronológico alguno. En vista de ello, resulta verdaderamente curioso que hasta 1853, coincidiendo con la publicación de la quinta edición de la obra ${ }^{4}$, la Academia y sus acólitos, como Núñez de Taboada (1825) y Salvá (1846), mantuvieron para dicha voz la marca que la identificaba como un arcaísmo; fecha a partir de la cual la misma Academia y, a partir de ella, Zerolo (1895), Alemany (1917), Pagés (1931) y María Moliner (1970), ${ }^{5}$ coinciden en su supresión. Por lo tanto, no sería descabellado presuponer que detrás del cambio en la consideración cronológica del presunto participio activo estaba el respeto y credibilidad que la obra de Domínguez había alcanzado, cuyo extraordinario éxito la convirtió en el segundo diccionario — después del académico — con mayor número de reediciones a lo largo del XIX (Seco 2003b: 293-297) y en una nueva fuente para el $D R A E$.

\section{UNA PALABRA EN BUSCA DE AUTOR}

Si tenemos en cuenta que la documentación que poseemos con relación a trocante es más bien escasa, como se comprobará inmediatamente, tampoco resultan demasiado evidentes los motivos que impulsaron su ingreso en el Diccionario usual. Podría pensarse en su presencia en el Universal vocabulario de Alonso de Palencia, en cuya parte romance encontramos:

Tamar: se interpreta por los varones muertos, e se interpreta trocante porque se transmudó en hábito de mundaria quando se echó con su suegro. Fue nuera de Judas, fijo de Jacob. También se interpreta amargura (1967 [1490]: s. v.; la cursiva es mía).

Se trata del primer y único testimonio fidedigno del término analizado, ${ }^{6}$ con el que se pretende trasladar al romance un commutans que aparece en la columna latina del

${ }^{4}$ Entre los fondos bibliográficos de la RAE, figuran las ediciones de 1848-1849, 1853, 1875 y 1878 del diccionario del romántico orensano. Aunque desconozco los verdaderos motivos, supongo que el primer ejemplar que se adquirió para la Academia, o el que se encontraba en un mejor estado de conservación (dado que también ha sido el utilizado para la confección del NTLLE), fue el de 1853. El hecho de que en la biblioteca de la institución sólo pueda consultarse una fotocopia del mismo — no así en el caso de la edición del 1848-1849—, dado que el original se guarda con celo, no hace sino confirmar mis sospechas. De ahí que la frontera temporal, el antes y el después de la desaparición de la marca ant. para trocante, se sitúe precisamente en dicha fecha.

${ }^{5}$ Todavía en la tercera edición del DUE (2007) se recoge trocante, si bien definido como sinónimo del adjetivo trocador 'aplicable al que trueca'.

${ }^{6}$ El evidenciado por el CORDE en primer lugar, perteneciente a una carta de cambio de 1461 («por quanto me distes e trocantes, e distes en troque e cambio por ello el terçio de la casa de Otero con sus vinnas»), es un error evidente que se coló en la versión electrónica del texto, ya que en la original, en papel, aparece claramente «distes e trocastes» (Álvarez Llopis 1994: 254). 
vocabulario. ${ }^{7}$ Efectivamente, como se narra en el Génesis 38, Tamar, viuda de Er y de su hermano Onán, hijos de Judá, «depositis viduitatis vestibus, assumpsit theristrum : et mutato habitu [...]» (Gen 38, 14), es decir, cambió sus ropas de luto por un vestido de verano y, disfrazada de esta guisa para no ser reconocida, mantuvo relaciones sexuales con su propio suegro, que la tomó por una prostituta, para asegurar su descendencia. Así, pues, Palencia optó por trasladar el participio latino, no con un cultismo crudo del tipo *conmutante, opción plausible a todas luces, ${ }^{8}$ sino con la raíz castellana patrimonial, o aquélla que se sentía como tal: trocar. Él mismo es garante de tal situación, puesto que la consideró como forma más adecuada para traducir cambire (s. v. cambio) y commutare. ${ }^{9}$

A pesar de ello, el vocablo empleado por el lexicógrafo humanista no pasó de ser una creación puntual $\mathrm{y}$, a lo que parece, un hápax que los académicos del siglo XVIII no consideraron, dado que el Universal vocabulario, tal vez por su estructura o por la forma en que son presentados los materiales, no fue elegido como fuente para documentar las voces contenidas en el Diccionario de Autoridades (en adelante $D A$ ) y, por ende, en el resto de ediciones del $D R A E$, que tomaron como base el primitivo lemario; de hecho, continúa siendo una de las ausencias más destacables en el NTLLE. Por ello, parece que hay que descartar su vinculación con el trocante de 1803.

Tuvieron que transcurrir casi trescientos años - doscientos ochenta y uno, en concretopara que se nos ofreciera una nueva documentación del término en cuestión. La oportunidad nos la ofrece la propia Academia, que en su primera Gramática de la lengua castellana (en adelante $G R A E$ ), al tratar de la definición y división del participio, advertía:

No de todos los verbos se pueden formar participios activos usuales: v. g. de los verbos trocar, perder, sufrir salen trocante, perdiente, sufriente, pero no los permite el uso. Ni todos los que pueden formarse de los verbos se deben considerar como verdaderos participios activos, porque son muy pocos los que conservan el régimen de sus verbos, $\mathrm{y}$ han pasado a ser adjetivos verbales que también suelen usarse como nombres sustantivos. Serían participios activos si pudiese decirse: causante la discordia: leyente los libros: oyente el sermon: porque este es el régimen de los verbos causar, leer, oir; pero careciendo de esta propiedad sus participios, vienen a quedar adjetivos verbales (GRAE: 178).

No importa tanto en esta ocasión subrayar la veracidad gramatical de las afirmaciones realizadas sobre la desaparición del participio de presente latino en español y su relación con

${ }^{7}$ «Tamar: interpretatur pro viris mortuis, eadem et commutans interpretatur, commutavit enim se in habitu meretricis quando cum socero suo concubuit. Nurus fuit Jude. Interpretatur quoque amaritudo». Palencia, o el texto por él seguido, no es más que un eco de Isidoro de Sevilla: «Thamar amaritudo pro viris mortuis. Item et commutans. Mutavit enim se in habitu meretricis, quando cum socero suo concubuit» (Etymologiarum, lib. VII, cap. VI, n. 39; Sevilla, 2004: 646). La fuente del hispalense pudo ser, a su vez, san Agustín (Contra faustum manicheum, lib. XXII, cap. LXXXVI; Patrología Latina 42, 457).

${ }^{8}$ Posibilidad que el mismo autor conocía, si bien adscrita al ámbito del comercio, pues que en la voz nomos se asevera que «nummus lex habetur inter commutantes», afirmación que fue traducida como «el dinero se toma por ley entre los que contratan». De nuevo parece evitarse el latinismo.

${ }^{9}$ «Solebant antiqui dicere campso campsas ut certo, pugno, inchoo, vel flecto latenter aufero, modo autem cambire dicimos pro commutare. [...] Los antiguos solían dezir campso, sas, por contiendo, lidio, comienço o doblego e quito ascondidamente, mas agora cambire dezimos por trocar uno por otro» (1967 [1490]: s. v. cambio). «Commutare: cum aliquo aliquid mutare, vendere. Commutare: es trocar algo con alguno e vender» (1967 [1490]: $s$. v. commutare). 
el postverbal -nte, cuanto la elección de los ejemplos con los que se ilustran dichos comentarios. Entre ellos, y en contraposición a formas adjetivas como sufriente o leyente, se incluye un perdiente, del que apenas se tienen testimonios, ${ }^{10} \mathrm{y}$ un trocante, cuya elección, debido a la ausencia de ejemplos, no sé si respondió a los designios del curioso azar. Su sola presencia en la primera gramática académica tal vez se tomara como una credencial de su existencia y un aval para su ingreso en el $D R A E$, aunque, si aceptáramos esta hipótesis, quedaría sin explicar el porqué tal recepción se produjo precisamente en 1803, y no en las tres primeras ediciones del diccionario $(1780,1783,1791)$, que vieron la luz entre la publicación de la GRAE y aquella fecha. ${ }^{11}$

Un tercer y definitivo indicio es el ofrecido por Esteban de Terreros, que en su Diccionario castellano con las voces de ciencias y artes (1786-1788) recoge la voz trocante, aunque adscribiéndola al ámbito de la cirugía y señalando que se trata de un 'instrumento de figura cónica que introducen candente en las cánulas, retirándole al punto'. Asimismo, en la cuarta acepción de trocar, vinculada al mismo campo especializado, se reenvía a troicar, que se define, a su vez, como 'instrumento de cirugía para agujerear la barriga'. Sin embargo, en el ámbito de la medicina no parece existir evidencia alguna de tales términos. ${ }^{12}$ Sí, en cambio, tenemos constancia de un trocar, sustantivo masculino de origen francés $(<$ fr. trois-quarts, con variantes troquart, trocart, trocar desde 1694; TLF: $s$. v. trocart $)^{13}$ que el propio jesuita recogió también en su diccionario, aseverando que, según los cirujanos, era un 'instrumento de plata o acero, en figura de aguja de cosa [sic: coser] de tres dedos de larga, con punta triangular metida en un cañuto'.

Efectivamente, lo que Terreros describía es un útil quirúrgico en forma de punzón, terminado en tres aristas cortantes, que, introducido en una cánula, sirve para perforar la pared de una cavidad del cuerpo y proceder a la evacuación de líquidos patológicos allí acumulados (cuando a finales del siglo XVIII se afirma que, además, se ponía al rojo vivo, imagino que se alude a cuestiones de asepsia). Una vez que la punción se ha realizado, se procede a la retirada

${ }^{10} \mathrm{El} C O R D E$ evidencia dos ocurrencias de la segunda mitad del siglo Xv, una de ellas procedente de Las Etimologías romanceadas de San Isidoro (p. 1450), donde perdens fidem es traducido como «perdiente la fe», y otra de la traslación del Strategematon de Sexto Julio Frontino (a. 1500).

${ }^{11}$ Curiosamente, también leyente, que cuenta con una abundante documentación a partir del siglo Xv, apareció recogido por primera vez en el $D R A E-1803$, lo que me indujo a sospechar en el aprovechamiento como autoridad léxica de la GRAE por la propia Academia.

${ }^{12}$ Agradezco a Bertha Gutiérrez Rodilla la inestimable ayuda que me ha prestado en la identificación del vocablo y en el esclarecimiento de su historia.

${ }^{13}$ Las propias y variadas formas francesas propiciaron la aparición, junto al oxítono trocar $(<\mathrm{fr}$. trocart), de una variante paroxítona trócar ( $<$ fr. trois-quarts), ya recogida en algunos repertorios como el de Zerolo (1895), Rodríguez Navas (1918), Pagés (1931) y en el Diccionario manual de 1989, que constituye un ejemplo aislado en la historia de la lexicografía académica. También en alemán, al menos por lo que se desprende de la información contenida en algunos repertorios léxicos, coexisten una pronunciación llana y otra aguda para trokar (Álvarez-Prada 1971: s. v.; Slabý 1989: s. v.). Son paroxítonas las formas inglesas trocar trochar trochart (OED: s. v. trocar), el italiano antiguo tro (a) carre (poco utilizado si lo comparamos al generalizado trequarti; $D E I$ : s. vv.) y el portugués troca(r)te (DELP: s. $v v$.), en estos dos últimos casos, por la adición de una -e paragógica. Frente a las opciones decimonónicas, y a causa del influjo de la lengua inglesa, en la moderna terminología médica española e italiana parecen preferirse las variantes llanas. En contraposición, trocánter 'protuberancia de la parte superior del fémur', que Terreros también recogió por primera vez como término quirúrgico — aunque más bien pertenece a la anatomíade origen francés, no ofreció ninguna duda acentual a los lexicógrafos posteriores, que la relacionaron siempre con su auténtica etimología griega $\tau \rho o \chi \alpha v \tau i ́ \rho$, convertida en llana tras su paso por el latín. 
del trocar para facilitar dicha extracción. ${ }^{14}$ De lo que parece que no se percató, sin embargo, es de que, bajo tres formas diferentes, cada una de ellas consideradas como lemas independientes, estaba definiendo un mismo objeto. Aunque el TLF no lo recoge, troicar, a simple vista, parece una variante gráfica de trois-quarts $>$ tro(i)car $(t)$, aspecto que parece confirmar el hecho de que el mismo autor le da como etimología un presunto francés homónimo. Por lo que se refiere a trocar, no existen dudas, dado que se identifica sin ningún problema con el fr. trocar o trois quarts y se ofrecen los equivalentes latinos acus triangularis chirurgicus y terebella (< TEREBRA 'trépano'). ${ }^{15}$ En cambio, en el caso de trocante, que bien pudiera ser fruto de una mala lectura, de un error ya presente en la fuente seguida o una acuñación del trucense, no se hace referencia a la utilidad quirúrgica del instrumento ${ }^{16}$ y se remite al fr. style 'punzón, estilete', lo que me lleva a pensar que el propio lexicógrafo albergaba algún tipo de duda con respecto al significante definido. Por lo tanto, se trataría de variantes o erratas que Terreros bien pudo haber conocido a través de los textos médicos y de los diccionarios franceses de los que se sirvió para la elaboración de su Diccionario castellano, ${ }^{17}$ aunque los tres vocablos aparecen allí recogidos sin el apoyo de ninguna autoridad expresa.

Una vez instalada en esta obra, la tríada de términos quedó a disposición, no sólo de los posibles usuarios del diccionario, sino también de otros lexicógrafos que le reservaron una estima y devoción muy particulares, dado que se configuraba como una de las primeras y más importantes recopilaciones del léxico científico y técnico. La Academia, como es sabido, incluyó en el $D A$ «las voces propias pertenecientes a artes liberales y mecánicas [...] más comunes y precisas al uso» (DA: Prólogo, 5), aplazando la elaboración de un vocabulario específico que, de todos es sabido, nunca llegó a materializarse. De ahí que el de Terreros comenzara a ser utilizado, por los miembros de la Corporación y por otros lexicógrafos, como fuente y testimonio de un buen número de voces especializadas. Así se explica, por ejemplo, que Salvá (1846) y la Biblioteca Ilustrada de Gaspar y Roig (1855) registraran el vocablo troicar, así como Zerolo (1895), que, como en el caso de trócar, la tomó como forma paroxítona: tróicar; todos ellos, no obstante, coincidieron en reconocerla como variante de trocar $\sim$ trócar.

Si Terreros se benefició del $D A$ para la redacción del suyo, los autores del DRAE se aprestaron igualmente a utilizar la información suministrada por el diccionario del jesuita como fuente y garante de ciertas nomenclaturas. En cuanto a la posible cronología de este último influjo, hace más de una década Jiménez Ríos señaló cómo, «una vez publicada y sacada a la luz toda la obra del jesuita, la corporación académica pudo introducir en su nueva edición, es decir, en la $4^{\mathrm{a}}$, publicada en 1803, algunos términos nuevos de las artes y de la técnica» (1996: 1367).${ }^{18} \mathrm{Y}$ es precisamente en dicha edición y en esa fecha cuando, como ya he advertido, apa-

${ }^{14}$ Desde entonces hasta hoy, dicho utensilio ha sufrido importantes cambios, particularmente relacionados con los materiales con los que se fabrica y con su forma, aunque continúa empleándose para los mismos usos. En Internet se encuentran numerosas fotografías y dibujos del mismo.

${ }^{15}$ Pocos años después, Manuel Hurtado de Mendoza afirmaba de trocar: «s. m. Terebellum vel Triquetrum. Se da este nombre a un instrumento de cirugía que consiste en un punzón de acero de unas dos pulgadas y media de largo, exactamente redondo, con un mango en su extremidad posterior, hecha a modo de una pera, y terminado por su extremidad anterior en una punta triangular ó de tres caras con tres ángulos cortantes de donde le viene ese nombre de trocar» (1840: $s$. v.).

${ }^{16}$ En el caso de trocar y troicar se menciona que sirve para abrir el vientre y para agujerear la barriga, respectivamente.

${ }^{17}$ Es posible consultar un elenco detallado de los mismos en Gutiérrez Rodilla (1996: 1330-1334).

${ }^{18}$ En las páginas precedentes del mismo trabajo se había hipotizado dicha relación en los siguientes términos: 
recen recogidos por primera vez en el $D R A E$ los sustantivos trocar y trocante. De modo que, tras la descripción realizada, es fácil deducir que en el $D R A E-1803$, a la zaga de Terreros, ambos términos fueron aceptados por la RAE como parte integral del léxico castellano, aunque se produjo una variación bastante significativa: trocante, debido a su forma y a la propia inseguridad que con respecto a ella se manifestaba en la obra que la había registrado por vez primera, sufrió una reinterpretación semántica. Los redactores se dejaron llevar por la predicción que aportan las RFP y consideraron el vocablo como el participio activo de trocar y, de acuerdo con tales premisas, lo definieron como 'el que trueca' e, incluso, lo equipararon el latino decipiens. Eso sí: dado que no conocían más testimonio que el Diccionario de Terreros, donde tampoco se ofrecía documentación alguna, se marcó como un uso anticuado, que en éste, como en tantos casos similares, parece significar uso desconocido.

Tras salir a la luz pública la cuarta edición del DRAE, trocante, como he señalado en el epígrafe segundo, se abrió paso y encontró acomodo en buena parte de la producción lexicográfica española, que confió una vez más en el poder de la letra impresa y en el respaldo que a tal término le otorgaba la Academia. Asimismo, cuando ésta dejó de considerarlo un uso arcaico, el resto de diccionarios volvieron a imitarla y eliminaron la marca ant. que hasta entonces lo había acompañado. La única y más importante diferencia es que, mientras que en el $D R A E$ 1992 se eliminó definitivamente, trocante continúa apareciendo en el resto de diccionarios, ${ }^{19} \mathrm{a}$ la espera de que algún incauto le conceda una existencia más allá del universo lexicográfico.

\section{CONCLUSIÓN}

Por lo tanto, trocante es un nuevo fantasma léxico que desde Terreros se ha transmitido de diccionario en diccionario. A la autoridad de éstos se sumó la credibilidad morfológica que aportaba el propio término, la cual, de acuerdo con los pronósticos que permiten aventurar las RFP, incluso avaló su inserción en la alternancia sufijal -nte/-dor. Sin embargo, su plausibilidad, su viabilidad lingüística, no puede ser un salvoconducto para su ingreso — así como el de tantos otros términos - en el $D R A E$, al menos hasta que no dispongamos de un repertorio histórico.

\section{REFERENCIAS BIBLIOGRÁFICAS}

Agustín, Obispo de Hipona (1980-1987): Sancti Aurelii Augustini Hipponensis Episcopi Opera Omnia, ed. de J. P. Migne, Turhout, Brepols (Patrología latina, vols. 32-47).

Álvarez Llopis, Elisa, Emma Blanco Campos y José Ángel García de Cortázar, eds. (1994): Colección diplomática de Santo Toribio de Liébana, Santander, Fundación Marcelino Botín.

Álvarez-PradA, Enrique y Gisela HABERKAMP DE ANTÓn (1971): Langenscheidt Handwörterbuch Spanisch, Berlin, Langenscheidt.

«En el caso de que la obra de Terreros hubiera influido en la actividad lexicográfica académica, este influjo se habría dado a partir de la $3^{\mathrm{a}}$ y $4^{\mathrm{a}}$ edición del $D R A E$, ya que habría que tener presente la fecha de distribución de cada uno de los tomos de aquel diccionario» (1996: 1360).

${ }^{19}$ Aparte del ya comentado caso del $D U E$, también en el $D E C H$ (s. v. trocar) se recoge trocante, aunque, claro está, sin aportar ninguna documentación. 
Buenafuentes de la Mata, Cristina y Carlos E. SÁnchez Lancis (2006): "Diccionario y morfología: la información morfológica en la 22. a edición del DRAE”, en M. Campos Souto y J. I. Pérez Pascual, coords., El Diccionario de la Real Academia Española: ayer y hoy, A Coruña, Universidade da Coruña, pp. 25-38.

CORDE: ReAl ACA DEMIA EsPa Ñola (30/04/2008): Corpus diacrónico del español, en línea: http://www.rae.es.

DA: Real Academia Española (2002 [1726-1739]): Diccionario de Autoridades, ed. facsímil, Madrid, Gredos.

DECH: Joan Corominas y José Antonio Pascual (1980-1991): Diccionario Crítico Etimológico Castellano e Hispánico, Madrid, Gredos.

DEI: Carlo Battisti y Giovanni Alessio (1950-1957): Dizionario etimologico italiano, Firenze, G. Barbera Editore.

DELP: José Pedro MaChado $\left(2003^{3}\right)$ : Dicionário etimológico da língua portuguesa, Lisboa, Livros Horizonte.

DRAE-2001: ReAl ACADEMIA Española (200122): Diccionario de la lengua española, Madrid, EspasaCalpe.

DUE: María Moliner (2007³): Diccionario de uso del español, Madrid, Gredos.

GRAE: ReAl Academia Española (1984 [1771]): Gramática de la lengua española, ed. facsímil, Madrid, Editora Nacional.

Gutiérrez Rodilla, Berta (1996): «El léxico de la medicina en el Diccionario de Esteban de Terreros y Pando», en A. Alonso et alii, eds., Actas del III Congreso Internacional de Historia de la Lengua Española, Madrid, Arco/Libros, II, pp. 1327-1342.

Hurtado de Mendoza, Manuel (1840): Vocabulario médico-quirúrgico o Diccionario de medicina y cirugía, Madrid, Boix.

JiMÉNEZ Ríos, Enrique (1996): «El diccionario de Terreros y las primeras ediciones del diccionario de la Academia», en A. Alonso et alii, eds., Actas del III Congreso Internacional de Historia de la Lengua Española, Madrid, Arco/Libros, II, pp. 1357-1369.

NTLLE: Real Academia Española (2001): Nuevo Tesoro Lexicográfico de la Lengua Española, Madrid, Espasa.

OED: James A. MurraY et alii, eds. (1978 [1933]): The Oxford English Dictionary, Oxford, Clarendon Press.

Palencia, Alonso de (1967 [1490]): Universal vocabulario en latín y en romance, ed. facsímil, Madrid, Comisión Permanente de la Asociación de Academias de la Lengua Española.

PenA, Jesús (2002): «Morfología derivativa y diccionario», en M. González Pereira, M. Souto y A. Veiga, coords., Léxico y gramática, Lugo, TrisTram, pp. 285-298.

Quirós GARcíA, Mariano (2007): «Haciendo y deshaciendo el diccionario: Léxico mercantil y acepciones fantasma en los tesoros de la Academia», Boletín de la Real Academia Española, LXXXVII, pp. 123-142.

SECO, Manuel (2003a): «El problema de la diacronía en los diccionarios generales», en Estudios de lexicografía española, Madrid, Gredos, 2. ${ }^{\text {e }}$ d. aumentada, pp. 70-80.

(2003b): «Un lexicógrafo romántico: Ramón Joaquín Domínguez», en Estudios de lexicografía

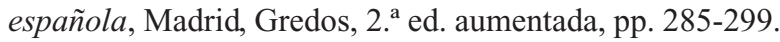


Sevilla, Isidoro de (2004): Etimologías, ed. de J. Oroz Reta y M.-A. Marcos Casquero, Madrid, BAC.

SlabÝ, Rodolfo J., Rodolfo Grossmann y Carlos Illig $\left(1994^{10}\right)$ : Diccionario de las lenguas española y alemana, Barcelona, Herder.

TERREROS, Esteban de (1987 [1786-1788]): Diccionario castellano con las voces de ciencias y artes, ed. facsímil, Madrid, Arco/Libros.

TlF: Centre National de la Recherche Scientifique (1971-1994): Trésor de la langue française. Dictionnaire de la langue du XIX et du XXe siècle (1789-1960), Paris, Gallimard.

Torres MARTínez, Marta (2006): «Algunos apuntes sobre morfología derivativa en las primeras obras académicas: Diccionario de Autoridades (1726-1739) y Gramática de la lengua castellana (1771)», en M. Villayandre, ed., Actas del XXXV Simposio Internacional de la Sociedad Española de Lingüística, León, Universidad de León, pp. 1847-1868. 